\title{
GONTINUED FRACTIONS WITH ABSOLUTELY CONVERGENT EVEN OR ODD PART
}

\author{
DAVID F. DAWSON
}

1. Introduction. The purpose of this paper is to give conditions under which the absolute convergence of the subsequence of odd or of even approximants to a continued fraction implies convergence of the continued fraction. In $\S 2$ we consider the problem in general, and in $\S 3$ we impose a condition which gives absolute convergence of the odd or of the even part of the continued fraction and state conditions which imply convergence of the continued fraction. If each of $a$ and $b$ is a complex number sequence, let $f(a)$ denote the continued fraction

$$
\frac{1}{1}+\frac{a_{1}}{1}+\frac{a_{2}}{1}+\frac{a_{3}}{1}+\ldots
$$

and $g(b)$ denote the continued fraction

$$
\frac{1}{b_{1}}+\frac{1}{b_{2}}+\frac{1}{b_{3}}+\ldots
$$

Let $f(a)$ have the approximants $f_{p}$ and $g(b)$ have the approximants $g_{p}$, where $f_{p}=A_{p} / B_{p}$ and $g_{p}=C_{p} / D_{p}$. Then

$$
\begin{aligned}
& A_{0}=0, A_{1}=1, A_{q+1}=A_{q}+a_{q} A_{q-1}, \\
& B_{0}=1, B_{1}=1, B_{q+1}=B_{q}+a_{q} B_{q-1}, \quad q=1,2,3, \ldots, \\
& C_{0}=0, C_{1}=1, C_{q+1}=b_{q+1} C_{q}+C_{q-1}, \\
& D_{0}=1, D_{1}=b_{1}, D_{q+1}=b_{q+1} D_{q}+D_{q-1}, \quad q=1,2,3, \ldots .
\end{aligned}
$$

If $b_{1}=1, a_{p} \neq 0,1 / b_{p+1}=a_{p} b_{p}, p=1,2,3, \ldots$, then (1.1) and (1.2) are equivalent in the sense that the two continued fractions have the same sequence of approximants. A well-known necessary condition for the convergence of $g(b)$ is that the series $\sum\left|b_{p}\right|$ diverge. Scott and Wall (2) investigated (1.1) by means of the systems of inequalities

$$
\begin{aligned}
& r_{1}\left|1+a_{1}\right| \geqq\left(1+r_{-1}\right)\left|a_{1}\right| \\
& r_{2 p+1}\left|1+a_{2 p}+a_{2 p+1}\right| \geqq r_{2 p+1} r_{2 p-1}\left|a_{2 p}\right|+\left|a_{2 p+1}\right|, \quad p=1,2,3, \ldots,
\end{aligned}
$$

and

$$
r_{2 p}\left|1+a_{2 p-1}+a_{2 p}\right| \geqq r_{2 p} r_{2 p-2}\left|a_{2 p-1}\right|+\left|a_{2 p}\right|, \quad p=1,2,3, \ldots,
$$

Received February 26, 1958, and in revised form September 22, 1958. This paper is based on a dissertation submitted to The University of Texas and prepared under the direction of Professor H. S. Wall. 
where $r_{p}$ is a non-negative number, $p=-1,0,1,2, \ldots$ They showed that if the $r_{p}$ are subjected to certain restrictions (for example, $r_{p}=1, p=-1$, $0,1,2, \ldots)$, then either some $a_{p}=0$ and $f(a)$ converges, or else $a_{p} \neq 0$, $p=1,2,3, \ldots$, and the divergence of the series $\sum\left|b_{p}\right|$ is necessary and sufficient for the convergence of $f(a)$. Lane and Wall (1) arrived at the same conclusion with the only restrictions on the $r_{p}$ that $r_{-1}$ and $r_{0}$ be distinct from zero by showing that if the even and odd parts of $f(a)$ converge absolutely, then $f(a)$ converges if, and only if, the series $\sum\left|b_{p}\right|$ diverges. This result is a consequence of two theorems (1, p. 371), the first of which states that if the even and odd parts of $f(a)$ are convergent, the even (odd) part being absolutely convergent, and if the series $\sum\left|h_{2 p-1}\right|$ (the series $\sum\left|h_{2 p}\right|$ ) diverges, then $f(a)$ converges. Here

$$
h_{p}=\frac{1}{1+a_{p+1} h_{p+1}}, \quad \quad p=1,2,3, \ldots
$$

The second of these theorems states that if there is a number $M$ such that $\left|f_{p}\right| \leqq M, p=1,2,3, \ldots$, and no term of $a$ is zero, then the two series $\sum\left|h_{p}\right|$ and $\sum\left|b_{p}\right|$ converge or diverge together. The question arises as to what restriction can be placed on the sequence $b$ which would replace the condition that the series $\sum\left|h_{2 p-1}\right|$ diverge in the first of these theorems. To answer this question by studying the relationships between the $b_{p}$ and $h_{p}$ appears difficult since the relationships are complicated:

$$
\begin{aligned}
& b_{1}=1, \quad b_{2}=-h_{1}, \quad b_{3}=-h_{2} \cdot \frac{1}{1-h_{1}}, \\
& b_{2 p+2}=-h_{2 p+1} \cdot \frac{\left(1-h_{1}\right)\left(1-h_{3}\right) \ldots\left(1-h_{2 p-1}\right)}{\left(1-h_{2}\right)\left(1-h_{4}\right) \ldots\left(1-h_{2 p}\right)}, \quad p=1,2,3, \ldots \\
& b_{2 p+3}=-h_{2 p+2} \cdot \frac{\left(1-h_{2}\right)\left(1-h_{4}\right) \ldots\left(1-h_{2 p}\right)}{\left(1-h_{1}\right)\left(1-h_{3}\right) \ldots\left(1-h_{2 p-1}\right)},
\end{aligned}
$$

We answer this question in $\S 2$ by studying the continued fraction (1.2). The result is that if $\left\{g_{2 p-1}\right\}\left(\left\{g_{2 p}\right\}\right)$ converges absolutely and $\left\{g_{2 p}\right\}\left(\left\{g_{2 p-1}\right\}\right)$ converges, then $g(b)$ converges, provided the series $\sum\left|b_{2 p-1}\right|$ (the series $\sum\left|b_{2 p}\right|$ ) diverges. This result is stronger than expected in the light of results stated above. We use this result to construct a simple proof of a theorem of Van Vleck (3): If there exists a positive number $k$ such that $\left|\operatorname{Im} b_{p}\right| \leqq k \cdot \operatorname{Re} b_{p}, p=1,2,3, \ldots$, and $b_{1} \neq 0$, then $g(b)$ converges if the series $\sum\left|b_{p}\right|$ diverges.

In $\S 3$ we turn our attention to the systems of inequalities (1.5) and (1.6). The two main results obtained are:

(1) If $(1.6)((1.5))$ holds, $0<r_{2 p-2} \leqq 1\left(0<r_{2 p-3} \leqq 1\right), p=1,2,3, \ldots$, and there exists a positive number $M$ such that

$$
M \leqslant \prod_{i=1}^{n} r_{2 i-2}\left(M \leqslant \prod_{i=1}^{n} r_{2 i-3}\right), \quad n=1,2,3, \ldots,
$$


then $f(a)$ converges, provided $a$ contains no zero term and the series $\sum\left|b_{2 p-1}\right|$ (the series $\sum\left|b_{2 p}\right|$ ) diverges;

(2) If (1.6) $((1.5))$ holds and there exists a number $r$ such that $0<r_{2 p-2}$ $\leqq r<1\left(0<r_{2 p-3} \leqq r<1\right), p=1,2,3, \ldots$, then $f(a)$ converges absolutely, provided $a$ contains no zero term. Several examples are given in connection with these results.

2. Convergence of $g(b)$. Here we study conditions which give convergence of $g(b)$ when one of the sequences $\left\{g_{2 p-1}\right\}$ and $\left\{g_{2 p}\right\}$ converges absolutely. Throughout this section, theorems are stated and proofs are given for the case that $\left\{g_{2 p-1}\right\}$ converges absolutely; the results for the other case follow by similar arguments.

LEMMA 2.1. If $z$ is a complex number sequence and there exists a non-negative integer $N$ such that the series

$$
\sum\left|1-\frac{z_{N+p+1}}{z_{N+p}}\right|
$$

converges, then z converges absolutely.

Proof. If $i>N$, then

$$
\exp \left|1-\frac{z_{i+1}}{z_{i}}\right| \geqslant 1+\left|\frac{z_{i+1}}{z_{i}}-1\right| \geqslant\left|\frac{z_{i+1}}{z_{i}}\right|-1+1=\left|\frac{z_{i+1}}{z_{i}}\right| .
$$

Hence, if $n$ is a positive integer, then

$$
\begin{aligned}
\left|\frac{z_{N+n+1}}{z_{N+1}}\right| & =\prod_{p=N+1}^{N+n}\left|\frac{z_{p+1}}{z_{p}}\right| \leqslant \prod_{p=N+1}^{N+n} \exp \left|1-\frac{z_{p+1}}{z_{p}}\right| \\
& =\exp \sum_{p=N+1}^{N+n}\left|1-\frac{z_{p+1}}{z_{p}}\right| \leqslant M,
\end{aligned}
$$

where

$$
M=\exp \sum_{p=N+1}^{\infty}\left|1-\frac{z_{p+1}}{z_{p}}\right|
$$

thus $\left|z_{N+n+1}\right| \leqq M\left|z_{N+1}\right|$. Therefore, there exists a number $k$ such that $\left|z_{p}\right|<k$, $p=1,2,3, \ldots$ Thus if $q$ is an integer greater than $N+1$, then

$$
\sum_{p=N+1}^{q}\left|z_{p}-z_{p+1}\right|=\sum_{p=N+1}^{q}\left|z_{p}\right| \cdot\left|1-\frac{z_{p+1}}{z_{p}}\right| \leqslant k \cdot \sum_{p=N+1}^{q}\left|1-\frac{z_{p+1}}{z_{p}}\right| .
$$

Therefore, $z$ converges absolutely.

THeOREM 2.1. If $\left\{g_{2 p-1}\right\}$ converges absolutely to $v$ and the series $\sum\left|b_{2 p-1}\right|$ diverges, then there is an infinite subsequence of $\left\{g_{2 p}\right\}$ which converges to $v$.

Proof. Suppose $\left\{g_{2 p-1}\right\}$ converges absolutely to $v$, the series $\sum\left|b_{2 p-1}\right|$ diverges, and $\left\{g_{2 p}\right\}$ contains no infinite subsequence which converges to $v$. There exists an integer $S$ such that if $p>S$, then $D_{2 p-1} \neq 0$. If $p>S$ and $D_{2 p} \neq 0$, then 


$$
\left|g_{2 p-1}-g_{2 p}\right|=1 /\left|D_{2 p-1} D_{2 p}\right| .
$$

Hence there exists a number $k$ such that, if $n$ is a positive integer, then $\left|D_{n+1} D_{n}\right|<k$, since $\left\{g_{2 p}\right\}$ contains no infinite subsequence which converges to $v$. Suppose $p>S$. Then

$$
\left|g_{2 p+1}-g_{2 p-1}\right|=\frac{\left|b_{2 p+1}\right|}{\left|D_{2 p+1} D_{2 p-1}\right|} .
$$

If there exists a number $R$ such that $\left|D_{2 p+1} D_{2 p-1}\right|<R, p=1,2,3, \ldots$, then if $n>S$ and $m$ is a positive integer, it follows that

$$
\sum_{p=n}^{n+m}\left|g_{2 p+1}-g_{2 p-1}\right|=\sum_{p=n}^{n+m} \frac{\left|b_{2 p+1}\right|}{\left|D_{2 p+1} D_{2 p-1}\right|} \geqslant \frac{1}{R} \sum_{p=n}^{n+m}\left|b_{2 p+1}\right| \text {. }
$$

But this contradicts the fact that the series $\sum\left|b_{2 p-1}\right|$ diverges. Thus if $R>0$, there exists a positive integer $i$ such that $\left|D_{2 i+1} D_{2 i-1}\right|>R$, and so either $\left|D_{2 i+1}\right|>R^{\frac{1}{2}}$ or $\left|D_{2 i-1}\right|>R^{\frac{1}{2}}$. Hence $\left\{D_{2 p-1}\right\}$ contains an unbounded subsequence. From (2.1) it follows that if $p>S$ and $D_{2 p} \neq 0$, then

$$
\left|g_{2 p+1}-g_{2 p-1}\right|=\frac{\left|b_{2 p+1}\right|\left|D_{2 p}\right|^{2}}{\left|D_{2 p+1} D_{2 p}\right|\left|D_{2 p} D_{2 p-1}\right|}
$$

and so, by (1.4),

$$
\left|1-\frac{D_{2 p+1}}{D_{2 p-1}}\right|=\frac{\left|b_{2 p+1} D_{2 p}\right|}{\left|D_{2 p-1}\right|}=\frac{\left|b_{2 p+1}\right|\left|D_{2 p}\right|^{2}}{\left|D_{2 p} D_{2 p-1}\right|}
$$

and

$$
\left|g_{2 p+1}-g_{2 p-1}\right|=\left|1-\frac{D_{2 p+1}}{D_{2 p-1}}\right| \cdot \frac{1}{\mid \overline{D_{2 p+1} D_{2 p} \mid}} ;
$$

which means that

$$
\left|1-\frac{D_{2 p+1}}{D_{2 p-1}}\right|=\left|D_{2 p+1} D_{2 p}\right|\left|g_{2 p+1}-g_{2 p-1}\right| \leqslant k\left|g_{2 p+1}-g_{2 p-1}\right| .
$$

On the other hand, if $p>S$ and $D_{2 p}=0$, then by (1.4), $D_{2 p+1}=D_{2 p-1}$, and so

$$
\left|1-\frac{D_{2 p+1}}{D_{2 p-1}}\right|=0
$$

Hence the series

$$
\sum\left|1-\frac{D_{2(S+p)+1}}{D_{2(S+p)-1}}\right|
$$

converges. Therefore, by the lemma, the sequence $\left\{D_{2 p-1}\right\}$ converges absolutely. But this contradicts a statement proved above that $\left\{D_{2 p-1}\right\}$ contains an unbounded subsequence. Therefore, our assumption that $\left\{g_{2 p}\right\}$ contains no infinite subsequence which converges to $v$ is proved false, and the theorem is established. 
THEOREM 2.2. If $\left\{g_{2 p-1}\right\}$ converges absolutely, $\left\{g_{2 p}\right\}$ converges, and the series $\sum\left|b_{2 p-1}\right|$ diverges, then $g(b)$ converges. There exists a sequence a such that the odd part of $f(a)$ converges absolutely, the even part converges, and the series $\sum\left|b_{p}\right|$ diverges, and $f(a)$ does not converge.

Proof. The first part of the theorem follows immediately from Theorem 2.1. We now construct a sequence $a$ as in the second part of the theorem. Let

$$
\begin{aligned}
f_{2 p-1} & =1 / 2^{p-1} \\
f_{2 p} & =\left\{\begin{array}{ll}
\frac{p+2}{p+3} & \text { if } p \text { is odd, } \\
\frac{p+3}{p+2} & \text { if } p \text { is even, }
\end{array} \quad p=1,2,3, \ldots .\right.
\end{aligned}
$$

If $p$ is a positive integer, let $a_{p+2}$ be the number determined by

$$
-a_{p+2}\left(f_{p}-f_{p+2}\right)\left(f_{p+1}-f_{p+3}\right)=\left(f_{p}-f_{p+1}\right)\left(f_{p+2}-f_{p+3}\right),
$$

and let $a_{1}=\frac{1}{3}$ and $a_{2}=-\frac{2}{3}$. Then $\left\{f_{p}\right\}_{p=1}^{\infty}$ is the sequence of approximants of $f(a)$ (5). Clearly $f(a)$ does not converge, but the odd part of $f(a)$ converges absolutely while the even part converges, but not absolutely. We note that $\left|a_{2} / a_{1}\right|=2$. Let $p$ be a positive integer. Then

$$
\frac{a_{2 p+2}\left(f_{2 p}-f_{2 p+2}\right)\left(f_{2 p+1}-f_{2 p+3}\right)}{a_{2 p+1}\left(f_{2 p-1}-f_{2 p+1}\right)\left(f_{2 p}-f_{2 p+2}\right)}=\frac{\left(f_{2 p}-f_{2 p+1}\right)\left(f_{2 p+2}-f_{2 p+3}\right)}{\left(f_{2 p-1}-f_{2 p}\right)\left(f_{2 p+1}-f_{2 p+2}\right)},
$$

and so

$$
\begin{aligned}
\left|\frac{a_{2 p+2}}{a_{2 p+1}}\right| & =\left|\frac{f_{2 p-1}-f_{2 p+1}}{f_{2 p+1}-f_{2 p+3}}\right|\left|\frac{f_{2 p}-f_{2 p+1}}{f_{2 p-1}-f_{2 p}}\right|\left|\frac{f_{2 p+2}-f_{2 p+3}}{f_{2 p+1}-f_{2 p+2}}\right| \\
& =2\left|\frac{f_{2 p}-f_{2 p+1}}{f_{2 p-1}-f_{2 p}}\right|\left|\frac{f_{2 p+2}-f_{2 p+3}}{f_{2 p+1}-f_{2 p+2}}\right| \\
& >2 .
\end{aligned}
$$

We note that if $p$ is a positive integer, then $\left|f_{2 p}-f_{2 p-1}\right|<2$, and so

$$
\begin{aligned}
\left|\frac{1}{a_{2 p+1}}\right| & =\left|\frac{\left(f_{2 p-1}-f_{2 p+1}\right)\left(f_{2 p}-f_{2 p+2}\right)}{\left(f_{2 p-1}-f_{2 p}\right)\left(f_{2 p+1}-f_{2 p+2}\right)}\right| \\
& >\frac{1}{4}\left|f_{2 p-1}-f_{2 p+1}\right|\left|f_{2 p}-f_{2 p+2}\right| \\
& =\frac{1}{2^{p+2}}\left|f_{2 p}-f_{2 p+2}\right| .
\end{aligned}
$$

Therefore, if $n$ is a positive integer, then

$$
\begin{aligned}
\left|b_{2 n+2}\right|=\frac{\left|\prod_{p=1}^{n} a_{2 p}\right|}{\left|\prod_{p=1}^{n+1} a_{2 p-1}\right|} & =\frac{1}{\left|a_{2 n+1}\right|} \prod_{p=1}^{n}\left|\frac{a_{2 p}}{a_{2 p-1}}\right| \\
& \geqslant 2^{n} \cdot \frac{1}{2^{n+2}}\left|f_{2 n}-f_{2 n+2}\right| \\
& =\frac{1}{4}\left|f_{2 n}-f_{2 n+2}\right| .
\end{aligned}
$$

Thus the series $\sum\left|b_{2 p}\right|$ diverges. This completes the proof of the theorem. 
We now use Theorem 2.2. in constructing a simple proof of the theorem of Van Vleck mentioned in the Introduction.

Theorem 2.3. (Van Vleck) If there exists a positive number $k$ such that

$$
\left|\operatorname{Im} b_{p}\right| \leqq k \cdot \operatorname{Re} b_{p}, \quad p=1,2,3, \ldots,
$$

and $b_{1} \neq 0$, then $g(b)$ converges provided the series $\sum\left|b_{p}\right|$ diverges.

Proof. Let

$$
t_{p}(u)=\frac{1}{b_{p}+u}, \quad \quad p=1,2,3, \ldots
$$

Then we see that if $p$ is a positive integer and $\operatorname{Re} u \geqq 0$, then $\operatorname{Re} t_{p}(u) \geqq 0$. Let $H$ denote the half-plane $z+\bar{z} \geqq 0$. If $n$ is a positive integer, let $T_{n}(u)$ $=t_{1} t_{2} \ldots t_{n}(u)$. We note that $T_{n}(H)$ is a circular disc and we denote its radius by $R_{n}$. We also note that $T_{p+1}(H)$ is a subset of $T_{p}(H), p=1,2,3, \ldots$. We find that

$$
T_{n}(u)=\frac{C_{n-1} u+C_{n}}{D_{n-1} u+D_{n}}
$$

and

$$
R_{n}=\frac{\frac{1}{2}}{\operatorname{Re} \bar{D}_{n} D_{n-1}}=\frac{\frac{1}{2}}{\sum_{n=1}^{n} \operatorname{Re} b_{p}\left|D_{p-1}\right|^{2}}
$$

Thus $T_{n}(0)=C_{n} / D_{n}=g_{n}$ and $T_{n}(\infty)=C_{n-1} / D_{n-1}=g_{n-1}$. Since $b_{n} \leqq$ $\operatorname{Re} b_{p}+\left|\operatorname{Im} b_{p}\right| \leqq \operatorname{Re} b_{p}+k \operatorname{Re} b_{p}=(1+k) \operatorname{Re} b_{p}, p=1,2,3, \ldots$, it follows that

$$
\begin{aligned}
(1+k) \operatorname{Re} \bar{D}_{n} D_{n-1} & =\sum_{p=1}^{n}(1+k) \operatorname{Re} b_{p}\left|D_{p-1}\right|^{2} \geqslant \sum_{p=1}^{n}\left|b_{p}\right|\left|D_{p-1}\right|^{2} \\
& \geqslant\left|\bar{D}_{n} D_{n-1}\right|, \quad n=1,2,3, \ldots
\end{aligned}
$$

Case 1. If $\operatorname{Re} \bar{D}_{n} D_{n-1} \rightarrow \infty$ as $n \rightarrow \infty$, then $R_{n} \rightarrow 0$ as $n \rightarrow \infty$ and $g(b)$ converges.

Case 2. If there exists a number $M$ such that $(1+k) \operatorname{Re} \bar{D}_{p} D_{p-1}<M$, $p=1,2,3, \ldots$, then if $m$ is a positive integer,

$$
\begin{aligned}
\sum_{p=1}^{m}\left|g_{p+1}-g_{p-1}\right| & =\sum_{p=1}^{m} \frac{\left|b_{p+1}\right|}{\left|D_{p+1} D_{p-1}\right|}=\sum_{p=1}^{m} \frac{\left|b_{p+1}\right|\left|D_{p}\right|^{2}}{\left|D_{p+1} D_{p}\right|\left|D_{p} D_{p-1}\right|} \\
& \leqslant L^{2} \sum_{p=1}^{m}\left|b_{p+1}\right|\left|D_{p}\right|^{2}<L^{2} M
\end{aligned}
$$

where $L=2 R_{1}$. Hence the even and odd parts of $g(b)$ converge absolutely. Thus, by Theorem $2.2, g(b)$ converges since either the series $\sum b_{2 p-1}$ or the series $\sum\left|b_{2 p}\right|$ diverges. Therefore the theorem is establisherl.

Remark 2.1. It is interesting to note in the above that actually $R_{n} \rightarrow 0$ as $n \rightarrow \infty$. If $c$ is a positive number, there exists a positive integer $V$, such that, if $n>N$, then 


$$
\left|g_{n}-g_{n-1}\right|=\frac{1}{\left|\bar{D}_{n} D_{n-1}\right|}<c
$$

and so

$$
\left|\bar{D}_{n} D_{n-1}\right|>\frac{1}{c}
$$

Thus

$$
\operatorname{Re} \bar{D}_{n} D_{n-1}>\frac{1}{c(1+k)}
$$

Therefore, $R_{n} \rightarrow 0$ as $n \rightarrow \infty$.

Remark 2.2. A curious corollary to Lemma 2.1 is that convergence of the series $\sum\left|C_{p} D_{p+1}\right|^{-1}$ implies convergence of the series $\sum\left|D_{p} D_{p+1}\right|^{-1}$. This is evident since, if $p$ is a positive integer,

$$
\left|1-\frac{g_{p+1}}{g_{p}}\right|=\left|\frac{g_{p}-g_{p+1}}{g_{p}}\right|=\frac{\left|C_{p} / D_{p}-C_{p+1} / D_{p+1}\right|}{\left|C_{p} / D_{p}\right|}=\frac{1}{\left|C_{p} D_{p+1}\right|} .
$$

Clearly, the converse is not true in general. For example, let $g_{p}=2^{-p}, p=1$, $2,3, \ldots$

3. Convergence of $f(a)$. Here we study conditions which give convergence of $f(a)$ when one of the systems of inequalities (1.5) and (1.6) holds. We shall state the theorems of this section in terms of the system (1.6) only. Similar results are obtained when (1.5) holds.

Theorem 3.1. If (1.6) holds, $r_{0} \neq 0$, and $a_{2 p} \neq 0, p=1,2,3, \ldots$, then $\left\{f_{2 p-1}\right\}$ converges absolutely to a point $v$ (a known result, (2, p. 155)), and if the series $\sum\left|a_{1} a_{3} \ldots a_{2 p-1}\right|\left|a_{2} a_{4} \ldots a_{2 p}\right|^{-1}$ diverges, then there exists an infinite subsequence of the sequence of approximants of the even part of $f(a)$ which converges to $v$.

Proof. From (1.6) and (1.3) we observe that

$$
r_{2 p}\left|B_{2 p+1}\right| \geqslant\left|a_{2 p}\right|\left|B_{2 p-1}\right|+\left(\prod_{i=0}^{p} r_{2 i}\right)\left|\prod_{i=1}^{p} a_{2 i-1}\right|, \quad p=1,2,3, \ldots
$$

By $(1.6), r_{2 p} \neq 0, p=1,2,3, \ldots$, since $a_{2 p} \neq 0, p=1,2,3, \ldots$, and from (3.1) it follows that $B_{2 p+1} \neq 0, p=1,2,3, \ldots$, (we note that $B_{1}=1$ ). Hence by (3.1),

$$
\begin{aligned}
& \left|f_{2 p+1}-f_{2 p-1}\right|=\frac{\left|a_{1} a_{2} \ldots a_{2 p-1}\right|}{\left|B_{2 p+1} B_{2 p-1}\right|} \\
& \quad \leqslant \frac{\left|a_{2} a_{4} \ldots a_{2 p-2}\right|}{r_{0} r_{2} \ldots r_{2 p-2}\left|B_{2 p-1}\right|}-\frac{\left|a_{2} a_{4} \ldots a_{2 p}\right|}{r_{0} r_{2} \ldots r_{2 p}\left|B_{2 p+1}\right|}, \quad p=2,3,4, \ldots .
\end{aligned}
$$

Therefore,

$$
\sum_{p=1}^{\infty}\left|f_{2 p+1}-f_{2 p-1}\right| \leqslant \frac{\left|a_{1}\right|}{\left|1+a_{1}+a_{2}\right|}+\frac{\left|a_{2}\right|}{r_{0} r_{2}\left|B_{3}\right|}
$$


and $\left\{f_{2 p-1}\right\}$ converges absolutely to a point $v$. We now require that the series

$$
\sum\left|a_{1} a_{3} \ldots a_{2 p-1}\right|\left|a_{2} a_{4} \ldots a_{2 p}\right|^{-1}
$$

diverge. Thus $a_{p} \neq 0, p=1,2,3, \ldots$ Hence we can consider the continued fraction (1.2) equivalent to $f(a)$. Since $\left|b_{2 p+1}\right|=\left|a_{1} a_{3} \ldots a_{2 p-1}\right|\left|a_{2} a_{4} \ldots a_{2 p}\right|^{-1}$ then by Theorem 2.1, the sequence of approximants of the even part of $f(a)$ contains an infinite subsequence convergent to $v$.

THEOREM 3.2. If (1.6) holds and there exists a positive number $M$ such that

$$
\begin{array}{ll}
0<r_{2 p-2} \leqslant 1, & p=1,2,3, \ldots, \\
M \leqslant \prod_{p=1}^{n} r_{2 p-2}, & n=1,2,3, \ldots,
\end{array}
$$

then $f(a)$ converges if either of the following conditions holds:

(i) $a_{p} \neq 0, p=1,2,3, \ldots$, and the series $\sum\left|b_{2 p-1}\right|$ diverges,

(ii) some $a_{2 p}=0$ and no $a_{2 p-1}=0$.

There exist a number sequence $\left\{r_{2 p}\right\}_{p=0}^{\infty}$ and a sequence a which satisfy (1.6), (1), and (i) such that $f(a)$ does not converge.

Proof. Suppose $a_{p} \neq 0, p=1,2,3, \ldots$ We consider the continued fraction (1.2) which is equivalent to $f(a)$, and note that

$$
\begin{aligned}
& B_{2 p}=a_{1} a_{3} \ldots a_{2 p-1} D_{2 p}, \\
& B_{2 p+1}=a_{2} a_{4} \ldots a_{2 p} D_{2 p+1},
\end{aligned} \quad p=1,2,3, \ldots
$$

From (3.1), (3.2), and (2), it follows that

$$
r_{2 p}\left|D_{2 p+1}\right| \geqq\left|D_{2 p-1}\right|+M\left|b_{2 p+1}\right|, \quad p=1,2,3, \ldots .
$$

Hence

$$
\left|D_{2 p+1}\right| \geqslant M \sum_{i=1}^{p+1}\left|b_{2 i-1}\right|
$$

since $D_{1}=1$ and $M \leqq 1$. If $n$ is a positive integer, then

$$
\begin{aligned}
& \left|g_{2 n+1}-g_{2 n}\right|=\frac{1}{\left|D_{2 n} D_{2 n+1}\right|}=\frac{\left|b_{2 n+1}\right|}{\left|D_{2 n+1}\right|\left|D_{2 n+1}-D_{2 n-1}\right|} \\
& \leqslant \frac{\left|b_{2 n+1}\right|}{\left|D_{2 n+1}\right|\left[\left|D_{2 n+1}\right|-\left|D_{2 n-1}\right|\right]} \leqslant \frac{1}{M\left|D_{2 n+1}\right|} \\
& \leqslant \frac{1}{M^{2} \sum_{p=1}^{n+1}\left|b_{2 p-1}\right|} \text {. }
\end{aligned}
$$

Therefore, since the odd part of $f(a)$ converges by Theorem 3.1, we see that $f(a)$ converges.

Suppose some $a_{2 q}=0$ and $a_{2 p-1} \neq 0, p=1,2,3, \ldots$ By (1.3) and (3.1) we see that $B_{p} \neq 0, p=0,1,2,3, \ldots$. Hence by a known theorem $(4, p .26)$, $f(a)$ converges. This completes the proof that $f(a)$ converges if either of the conditions (i) or (ii) holds. 
Let

$$
\begin{aligned}
& r_{2 p-2}=\left\{\begin{array}{l}
1 \text { if } p \text { is odd } \\
\frac{1}{2} \text { if } p \text { is even, }
\end{array}\right. \\
& a_{2 p-1}=a_{2 p}=\left\{\begin{array}{l}
1 \text { if } p \text { is odd } \\
-2 \text { if } p \text { is even, }
\end{array} \quad p=1,2,3, \ldots .\right.
\end{aligned}
$$

Then (1), (1.6), and (i) are satisfied and $f(a)$ does not converge since the sequence of approximants of the even part of $f(a)$ contains an infinite subsequence of zero terms while the odd part of $f(a)$ does not converge to zero. This completes the proof of the theorem.

Remark 3.1. In (ii) of Theorem 3.2, the condition that $a_{2 p-1} \neq 0, p=1,2$, $3, \ldots$, in case some $a_{2 p}=0$ cannot be removed. We see this in the following example: let $r_{2 p}=1, p=0,1,2, \ldots, a_{1}=a_{2}=1, a_{3}=a_{6}=0, a_{4}=a_{5}=-\frac{1}{2}$, $a_{p}=1, p=7,8,9, \ldots$ Clearly (1), (2), and (1.6) are satisfied. Simple calculations show that $B_{6}=B_{7}=0$, and so by $(1.3), B_{p}=0, p=6,7,8, \ldots$.

Remark 3.2. In Theorem 3.2, in case $a_{2 p} \neq 0, p=1,2,3, \ldots$, but some $a_{2 p-1}=0$, the continued fraction does not necessarily converge, as shown by the following example: let $r_{2 p}=1, p=0,1,2,3, \ldots, a_{1}=0$, and

$$
a_{2 p}=a_{2 p+1}=\left\{\begin{array}{l}
-\frac{1}{2} \text { if } p \text { is odd } \\
1 \text { if } p \text { is even, }
\end{array} \quad p=1,2,3, \ldots .\right.
$$

Then $B_{4 p}=0, p=1,2,3, \ldots$

THEOREM 3.3. If (1.6) holds and there exists a number $r$ such that $0<r_{2 p-2}$ $\leqq r<1, p=1,2,3, \ldots$, then $f(a)$ converges absolutely, provided one of the sequences $\left\{a_{2 p-1}\right\}$ and $\left\{a_{2 p}\right\}$ contains no zero term.

Proof. Suppose $a_{p} \neq 0, p=1,2,3, \ldots$ Again we consider the continued fraction (1.2) which is equivalent to $f(a)$. If $n$ is a positive integer, then

$$
\begin{aligned}
\left|g_{2 n+1}-g_{2 n}\right|=\frac{1}{\left|D_{2 n+1} D_{2 n}\right|} & =\frac{\left|b_{2 n+1}\right|}{\left|D_{2 n+1}\right|\left|D_{2 n+1}-D_{2 n-1}\right|} \\
& =\frac{\left|b_{2 n+1}\right|}{\left|D_{2 n+1} D_{2 n-1}\right|} \cdot \frac{1}{\left|D_{2 n+1} / D_{2 n-1}-1\right|} \\
& \leqslant\left|g_{2 n+1}-g_{2 n-1}\right| \cdot \frac{1}{1 / r_{2 n}-1} \\
& \leqslant\left|g_{2 n+1}-g_{2 n-1}\right| \cdot \frac{r}{1-r} .
\end{aligned}
$$

Thus it follows that $f(a)$ converges absolutely, since the odd part of $f(a)$ converges absolutely, as shown in the proof of Theorem 3.1.

Suppose some $a_{2 q}=0$ and $a_{2 p-1} \neq 0, p=1,2,3, \ldots$ Then by (3.1) and (1.3)

$$
\left|B_{2 p}\right|=\left|B_{2 p+1}-a_{2 p} B_{2 p-1}\right| \geqq\left|B_{2 p+1}\right|-\left|a_{2 p} B_{2 p-1}\right|>0, \quad p=1,2,3, \ldots
$$

Hence $B_{p} \neq 0, p=1,2,3, \ldots$ Therefore $f(a)$ converges absolutely (4, p. 26). 
On the other hand, suppose some $a_{2 q-1}=0$ and $a_{2 p} \neq 0, p=1,2,3, \ldots$. Since $r_{2 p} \leqq r<1, p=1,2,3, \ldots$, we see by (3.1) that

$$
\left|B_{2 p-1}\right|>\left|a_{2 p}\right|\left|B_{2 p-1}\right|, \quad \quad p=1,2,3, \ldots
$$

and so $B_{2 p-1} \neq 0, p=1,2,3, \ldots$, since $a_{2} B_{1} \neq 0$. Therefore, as before, $B_{p} \neq 0, p=1,2,3, \ldots$, and $f(a)$ converges absolutely.

Remark 3.3. We give an example of a number sequence $\left\{r_{2 p}\right\}_{p=0}{ }^{(1)}$ and an unbounded sequence $a$ which satisfy the hypothesis of Theorem 3.3 as follows: let $r_{2 p-2}=\frac{1}{2}, p=1,2,3, \ldots$, and $a_{1}=a_{2}=1, a_{2 p-1}=-\left(2^{p}\right)$, $a_{2 p}=-\left(2^{p-1}\right)+1, p=2,3,4, \ldots$

Remark 3.4. There exist a number sequence $\left\{r_{2 p}\right\}_{p=0}^{\infty}$ and a sequence $a$ such that all of the conditions of Theorem 3.3. are satisfied except that each of the sequences $\left\{a_{2 p-1}\right\}$ and $\left\{a_{2 p}\right\}$ contains a zero term and $f(a)$ does not converge. Let $r_{2}=\frac{1}{3}, r_{2 p}=\frac{1}{2}, p=0,2,3,4, \ldots$, and $a_{1}=a_{4}=0, a_{2}=-\frac{1}{4}$, $a_{3}=-\frac{3}{4}, a_{p}=1, p=5,6,7, \ldots$ Since $B_{4}=1+a_{1}+a_{2}+a_{3}\left(1+a_{1}\right)=0$ and $B_{5}=B_{4}+a_{4} B_{3}=0$, by $(1.3), B_{p}=0, p=4,5,6, \ldots$

\section{REFERENCES}

1. R. E. Lane and H. S. Wall, Continued fractions with absolutely convergent even and odd parts, Trans. Amer. Math. Soc., 67 (1949), 368-80.

2. W. T. Scott and H. S. Wall, A convergence theorem for continued fractions, Trans. Amer. Math. Soc., 47 (1940), 155-72.

3. E. B. Van Vleck, On the convergence of continued fractions with complex elements, Trans. Amer. Math. Soc., 2 (1901), 215-33.

4. H. S. Wall, Analytic theory of continued fractions (New York, 1948).

5. H. S. Wall, Continued fractions and cross-ratio groups of Cremona transformations, Bull. Amer. Math. Soc., 40 (1934), 578-92.

University of Missouri 